\title{
Properties of the Lunar Detection Mode for ZeV-Scale Particles with LOFAR
}

\author{
T. Winchen ${ }^{1, \star}$, A. Bonardi ${ }^{2}$, S. Buitink ${ }^{1}$, A. Corstanje $^{2}$, H. Falcke Fi,3, $^{2,3}$ B. M. Hare ${ }^{5}$, J. R. \\ Hörandel ${ }^{1,2,3}, P$. Mitra ${ }^{1}, K$. Mulrey ${ }^{1}, A$. Nelles ${ }^{2,6}, J$. P. Rachen ${ }^{2}$, L. Rossetto ${ }^{2}, P$. Schellart ${ }^{2,7}, O$. \\ Scholten $^{5,8}$, S. ter Veen ${ }^{2,4}$, S. Thoudam ${ }^{2,9}$, T. N. G. Trinh ${ }^{5}$ \\ ${ }^{1}$ Astrophysical Institute, Vrije Universiteit Brussel, Pleinlaan 2, 1050 Brussels, Belgium \\ ${ }^{2}$ Department of Astrophysics/IMAPP, Radboud University, P.O. Box 9010, 6500 GL Nijmegen, \\ The Netherlands \\ ${ }^{3}$ NIKHEF, Science Park Amsterdam, 1098 XG Amsterdam, The Netherlands \\ ${ }^{4}$ Netherlands Institute of Radio Astronomy (ASTRON), Postbus 2, 7990 AA Dwingeloo, The Netherlands \\ ${ }^{5}$ KVI-CART, University Groningen, P.O. Box 72, 9700 AB Groningen \\ ${ }^{6}$ Department of Physics and Astronomy, University of California Irvine, Irvine, CA 92697-4575, USA \\ ${ }^{7}$ Department of Astrophysical Sciences, Princeton University, Princeton, NJ 08544, USA \\ ${ }^{8}$ Interuniversity Institute for High-Energy, Vrije Universiteit Brussel, Pleinlaan 2, 1050 Brussels, Belgium \\ ${ }^{9}$ Department of Physics and Electrical Engineering, Linnéuniversitetet, 35195 Växjö, Sweden
}

\begin{abstract}
The steep decrease of the flux of ultra-high energy cosmic rays (UHECR) provides a challenge to answer the long standing question about their origin and nature. A significant increase in detector volume may be achieved by employing Earth's moon as a detector that is read out using existing Earth-bound radio telescopes by searching for the radio pulses emitted by the particle shower in the lunar rock. In this contribution we will report on the properties of a corresponding detection mode currently under development for the LOFAR Radio telescope.
\end{abstract}

\section{Introduction}

Among the major challenges in astroparticle physics are the low fluxes of the particles of interest which consequently require huge detectors at the highest energies. Already in the 1960s it had been proposed [1] to use the moon as a particle detector by searching for the ns radio pulses generated by a charge excess in the shower developing in the lunar rock. Several searches with earth-bound radio telescopes have been conducted since then (see reference [2] for a review) that have so far not been sensitive enough to detect the guaranteed flux of charged cosmic rays. These previous searches used telescopes operating at $\mathrm{GHz}$ frequencies, where the amplitude of the emitted pulse is at maximum. However, while the amplitude increases towards higher frequencies, the effective area increases towards lower frequencies as the Cherenkov cone becomes broader and thus also inclined events become detectable. Consequently, the optimum frequency band for corresponding observations is above approximately $100 \mathrm{MHz}$ [3].

^e-mail: tobias.winchen@ rwth-aachen.de; This work is funded by ERC grant 640130 and DFG grant WI 4946/1-1 
The LOw Frequency ARray (LOFAR) [4] is the currently largest radio telescope operating in this frequency range. LOFAR is composed of more than 50 stations located throughout Europe with 24 stations placed in a dense core in the Netherlands. Each of these core-stations contains 768 high band antennas (HBA) operating in the frequency range $110 \mathrm{MHz}$ to $190 \mathrm{MHz}$ organized in two fields of 24 tiles of 16 antennas each. The signals of the individual antennas of a station are filtered into sub-bands by a polyphase filter (PPF) combined into a beam of approximately $5^{\circ}$ width at $120 \mathrm{MHz}$ before it can be accessed by users of the telescope with dedicated observation pipelines.

\section{Design and Sensitivity of the Lunar Detection Pipeline}

The lunar detection pipeline [5-7] requires combining individual stations into a grid of tighter beams covering the moon, inversion of the PPF and correction for ionospheric dispersion. We aim for a realtime processing of the available data to trigger the readout of the buffered raw signal for offline analysis. The data processing will be done on the DRAGNET cluster [8] which provides the required computing performance with GPUs. However, due to limitations of its connection to the LOFAR network only data of five out of the 24 stations can be processed.

To estimate the expected sensitivity of the pipeline we simulated data traces by adding the signal expected from pulses of varying strength originating from the direction of observation to air shower triggered noise data. The simulated pulses are dispersed assuming an exact slant total electron content (STEC) of 20 TECU $\left(1 \mathrm{TECU}=10^{16} \mathrm{~m}^{-2}\right.$ ), respectively a normal distributed STEC value centered around $20 \mathrm{TECU}$ with width of 1 TECU to account for imperfect reconstruction. All traces were processed by the observation pipeline with an dedispersion corresponding to 20 TECU. As trigger condition we require one sample above a threshold value in at least one out of 49 traces for analysis beams forming a grid covering the moon. The threshold value is chosen to yield a trigger rate of $1 /$ min from thermal noise only. In figure 1 the resulting trigger efficiency is shown as function of the pulse amplitude. We expect full efficiency to pulses with amplitude $E \approx 0.8 \mu \mathrm{Vm}^{-1}$ and approximately $20 \%$ efficiency at $E \approx 0.2 \mu \mathrm{V} \mathrm{m}^{-1}$ if the STEC is known to 1 TECU.

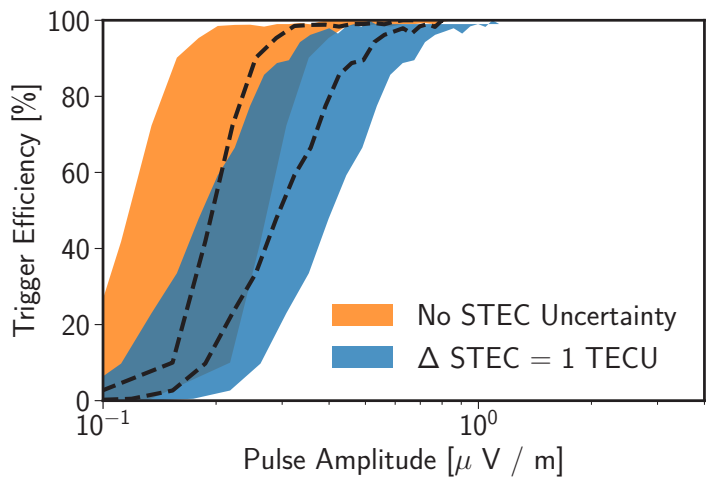

Figure 1. Trigger efficiency as function of pulse amplitude for simulated pulses added to measured background. Pulses have been dedispersed assuming an ionospheric electron content of 20 TECU and dispersed by the same amount (orange) respectively a random value of $20 \pm 1$ TECU (blue). The bands denote the uncertainty on the pulse amplitude given by the uncertainty of the antenna calibration.

Based on this trigger efficiency we calculated the sensitivity to cosmic particles shown in figure 2 following the procedure described in $[2,14]$, which is based on semi-analytical estimates for the particle interaction in the lunar rock and the amplitude for the escaping pulse. We only replaced the estimate for the minimum detectable field strength with the result of our simulations. Compared to previous estimates [2] we use a reduced trigger threshold and increased band-width which increases the sensitivity, but only use the signal of 5 out of 24 stations. Nevertheless, the net expected sensitivity is increased and in particular for charged particles the expected sensitivity approaches the flux observed by other experiments. 

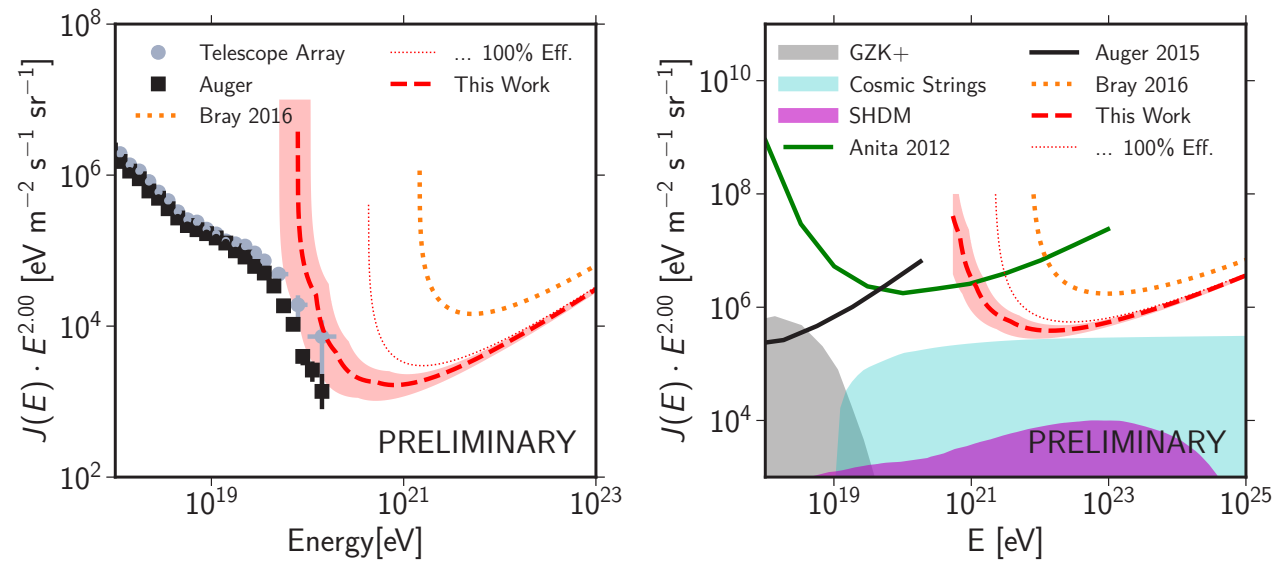

Figure 2. Observed fluxes and expected limits for UHECR (left) and neutrinos (right) with available data [9, 10] for the UHECR flux respectively model predictions for the flux of neutrinos [11-13] for 200 hours of observation. The dashed line labeled $100 \%$ Eff. corresponds to a minimum detectable field strength of $\mathrm{E}=0.8 \mu \mathrm{V} \mathrm{m} \mathrm{m}^{-1}$.

\section{Conclusion}

We have implemented a prototype for the online analysis chain for the lunar detection of $\mathrm{ZeV}$ particles with the LOFAR radio telescope including a full simulation of the telescope processing. This enables for the first time a sensitivity calculation for lunar observations to detect $\mathrm{ZeV}$ scale particles with LOFAR using a detector Monte Carlo. Compared to previous estimates based on semi-analytical calculations of the detector efficiency, the expected sensitivity increases, as also the regime below full efficiency can be considered. However, this estimate still relies on semi-analytical estimates for the interaction and pulse escape in the moon and is expected to improve in the future with detailed simulations.

\section{References}

[1] G. Askaryan, Sovjet Physics J.E.T.P 14, 441 (1962)

[2] J. Bray, Astroparticle Physics 77, 1 (2016)

[3] O. Scholten et al., Astroparticle Physics 26, 219 (2006)

[4] M.P. van Haarlem et al., Astronomy and Astrophysics 556, A2 (2013)

[5] K. Singh et al., Nucl. Instrum. Meth. A664, 171 (2012)

[6] T. Winchen et al., EPJ Web Conf. 135, 04003 (2017)

[7] T. Winchen et al. in Proceedings of the 35th ICRC (2017)

[8] J. Hessels, DRAGNET (2015), http://www . astron.nl/dragnet

[9] F. Fenu for the Pierre Auger Collaboration in Proceedings of the 35th ICRC (2017)

[10] D. Ivanov, Ph.D. thesis, Rutgers, The State University of New Jersey (2012)

[11] E. Roulet, G. Sigl, A. van Vliet, S. Mollerach, JCAP 1, 028 (2013)

[12] V. Berezinsky, E. Sabancilar, A. Vilenkin, Phys. Rev. D84, 085006 (2011)

[13] R. Aloisio, S. Matarrese, A.V. Olinto, JCAP 1508, 024 (2015)

[14] K.G. Gayley, R.L. Mutel, T.R. Jaeger, Astrophys. J. 706, 1556 (2009) 